Supporting Information

\title{
Template-Assisted Magnetron Sputtering of Cotton Nonwovens for Wound Healing Application
}

Shangpeng Liu ${ }^{\dagger \perp}$, Jiwei Li* ${ }^{* \dagger+}$, Shaohua Zhang ${ }^{\S}$, Xiying Zhang" , Jianwei Ma ${ }^{\dagger}, \mathrm{Na}$ Wang $^{\dagger}$, Shuang Wang $\$$, Bin Wang $\$$, Shaojuan Chen $* \dagger$

${ }^{\dagger}$ Industrial Research Institute of Nonwovens and Technical Textiles, College of Textiles and Clothing, Qingdao University, Qingdao, 266071, P. R. China.

$\$$ Department of Biochemistry and Microbiology, Qingdao University, Qingdao, 266071, P. R. China.

$\S$ Department of Pediatrics, the Affiliated Hospital of Qingdao University, Qingdao, 266003, P. R. China.

II Department of Pathology, the Second Hospital of Shandong University, Jinan, 250033, P. R. China

\section{Corresponding Author}

*jiweili@qdu.edu.cn (Jiwei Li)

* qdchshj@126.com (Shaojuan Chen) 

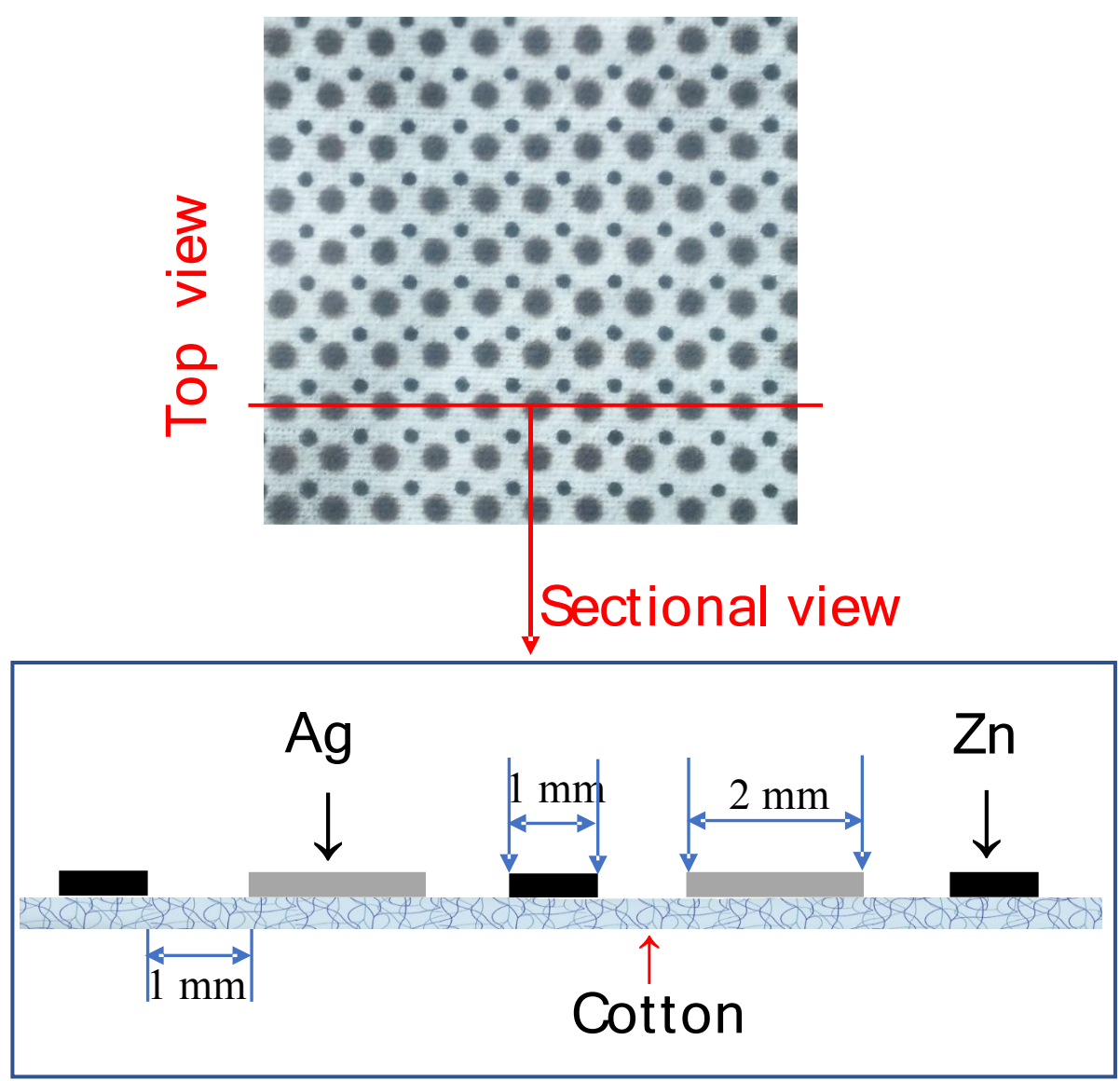

Figure S1 Size parameters of Ag/Zn@Cotton
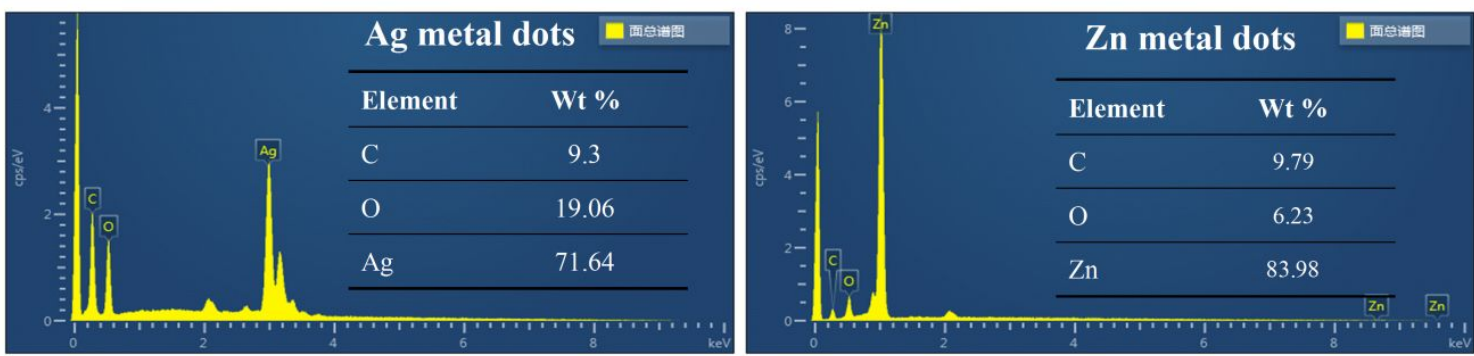

Figure S2 EDS analysis of the metal dots. 

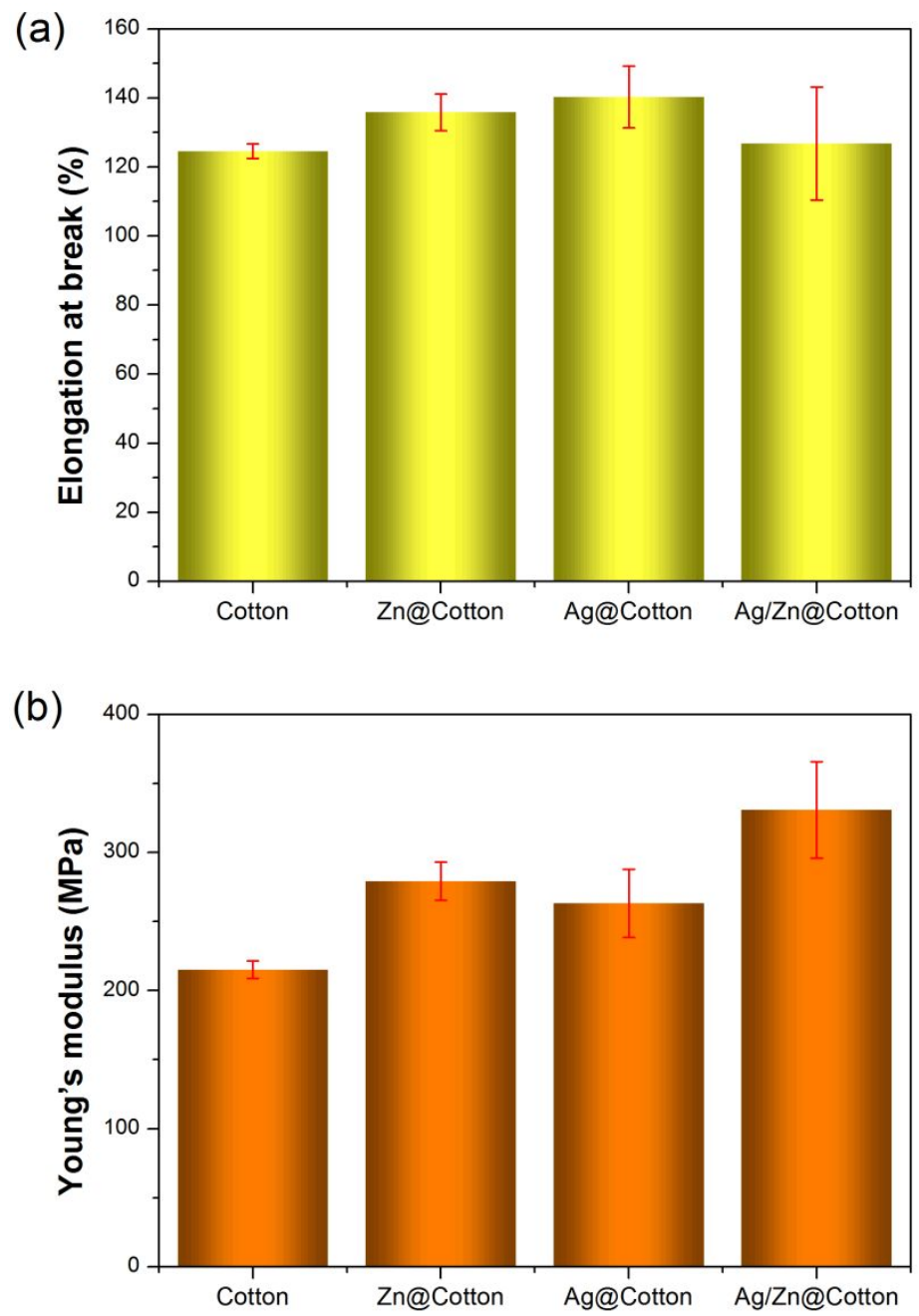

Figure S3 Elongation at break (a) and Young's modulus (b) of Cotton, Zn@Cotton, Ag@Cotton, and Ag/Zn@Cotton. 


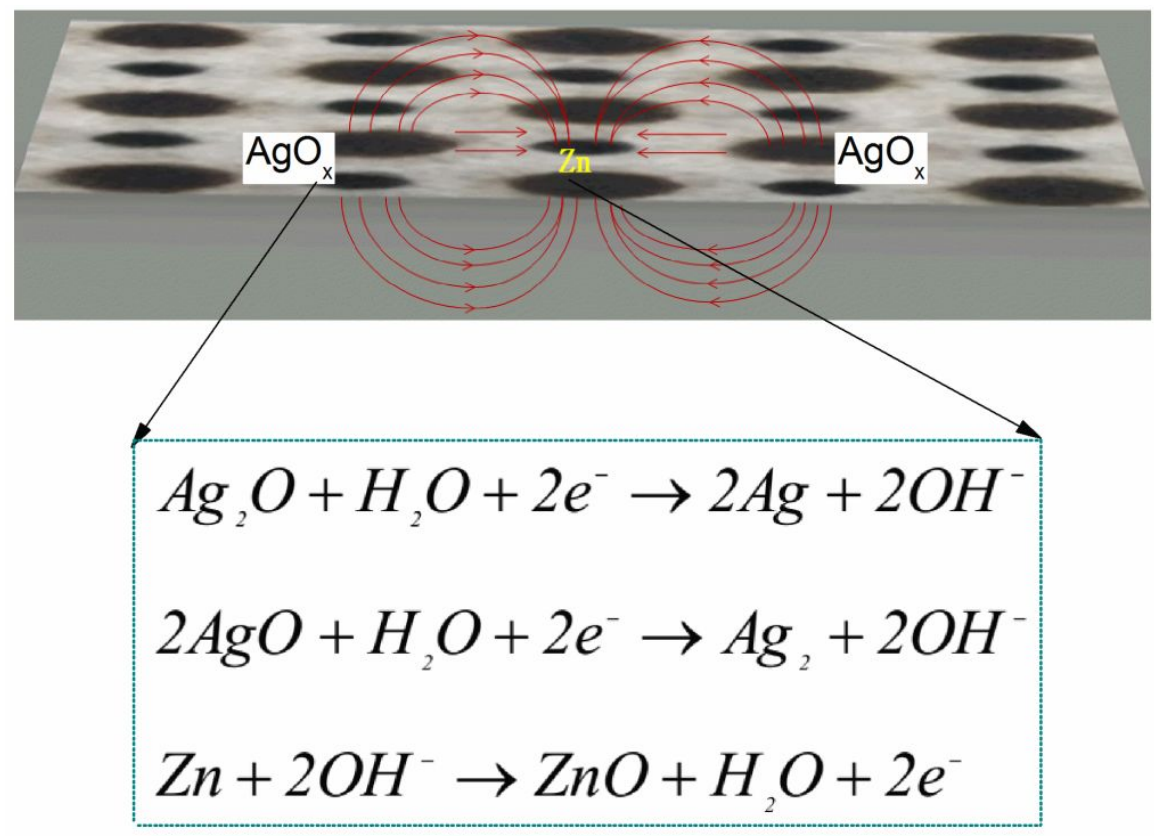

Figure S4 Schematic diagram of the electric fields generated by the Ag/Zn@Cotton and the electrode reaction. (The conducting fluid can be wound exudate, blood, and saline solution, etc.) 


\section{Ag@Cotton without template}

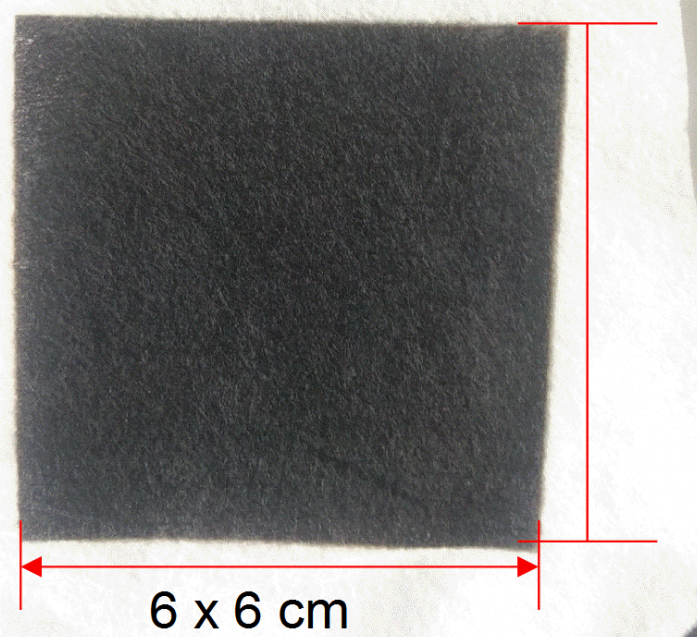

\section{Zn@Cotton without template}

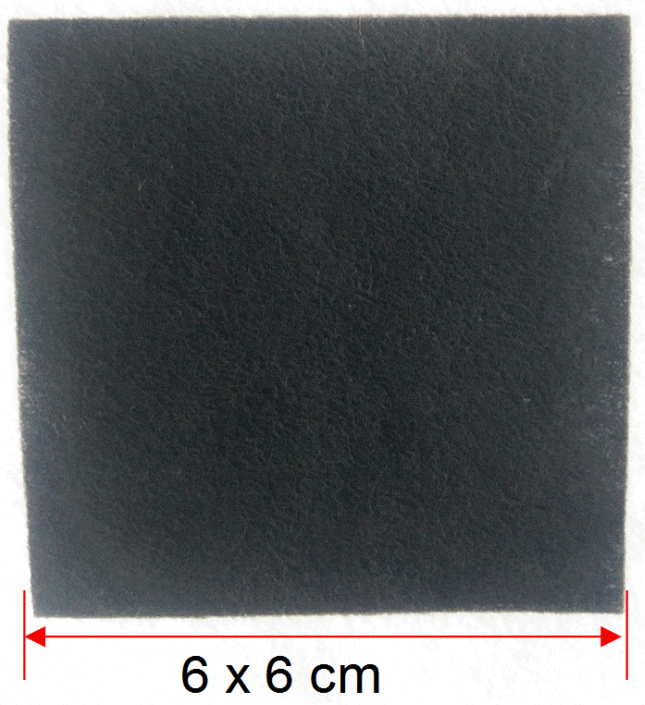

Figure S5 SEM digital image of the Ag@Cotton and Zn@Cotton without template. Since the anodic and cathodic dots on Ag@Cotton or Zn@Cotton are too small, the water contact angle test was performed on the Ag@Cotton and Zn@Cotton without template (Figure S3). 


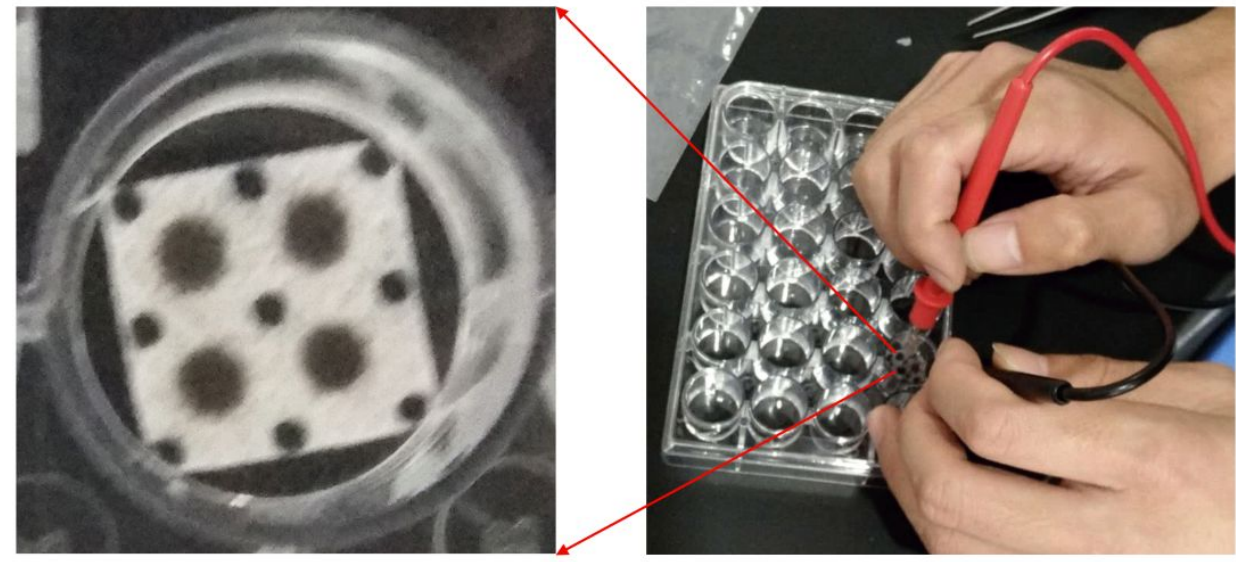

Ag/Zn@Cotton moistened by $0.9 \% \mathrm{NaCl}$

Figure S6 The photographs of the mearsurement of the voltage and current of Ag/Zn@Cotton.

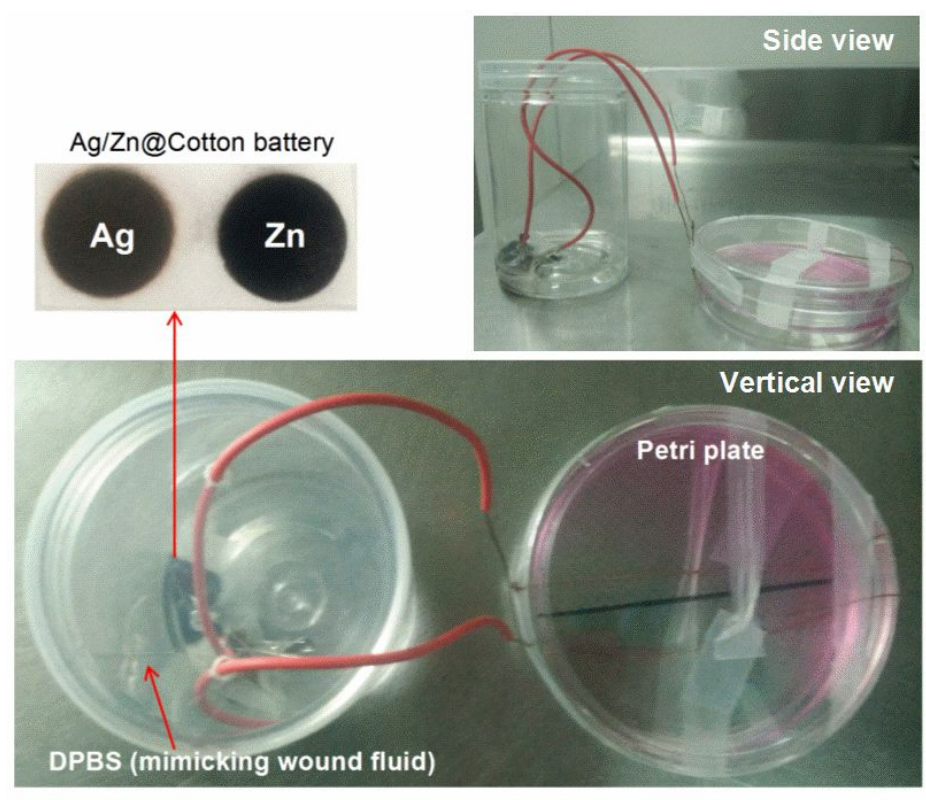

Electrical stimulation generation part Electrical cell culture plate

Figure S7 Diagram of the scratch wound assay device for the Ag/Zn@Cotton. 